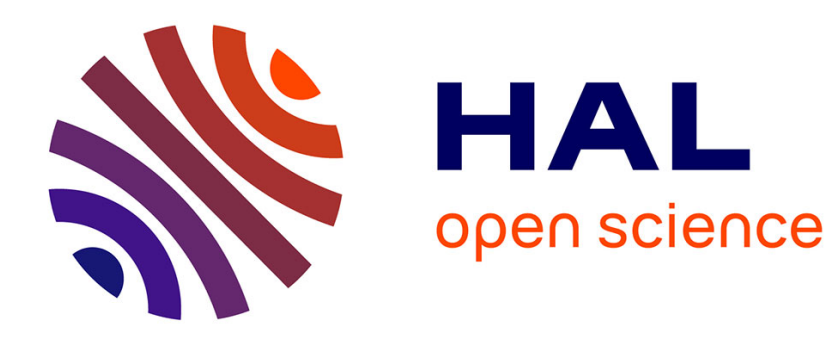

\title{
La dilatation thermique des alliages dilués $\mathrm{Cu}-\mathrm{Al}$ \\ J.P. Ganne
}

\section{To cite this version:}

J.P. Ganne. La dilatation thermique des alliages dilués Cu-Al. Journal de Physique Lettres, 1979, 40

(18), pp.481-483. 10.1051/jphyslet:019790040018048100 . jpa-00231670

\section{HAL Id: jpa-00231670 https://hal.science/jpa-00231670}

Submitted on 1 Jan 1979

HAL is a multi-disciplinary open access archive for the deposit and dissemination of scientific research documents, whether they are published or not. The documents may come from teaching and research institutions in France or abroad, or from public or private research centers.
L'archive ouverte pluridisciplinaire HAL, est destinée au dépôt et à la diffusion de documents scientifiques de niveau recherche, publiés ou non, émanant des établissements d'enseignement et de recherche français ou étrangers, des laboratoires publics ou privés. 


\title{
La dilatation thermique des alliages dilués $\mathrm{Cu}-\mathrm{Al}$
}

\author{
J. P. Ganne \\ Section d'Etude des Solides Irradiés, Centre d'Etudes Nucléaires de Fontenay-aux-Roses, 92260, France
}

(Reçu le 2 juillet 1979, accepté le 25 juillet 1979)

\begin{abstract}
Résumé. - Nous avons mesuré l'effet de l'aluminium en solution solide à des concentrations atomiques de 1 à $7 \%$ sur la dilatation thermique du cuivre. Nous trouvons une variation relative du coefficient de dilatation thermique $\Delta \alpha / \alpha c=-1,1 \times 10^{-3}$ par pour cent atomique d'aluminium de même signe, mais trois fois plus faible en valeur absolue que la valeur $-3,8 \times 10^{-3}$ calculée par Cain et Thomas à partir de mesures de constantes élastiques du $2^{\mathrm{e}}$ et $3^{\mathrm{e}}$ ordre.
\end{abstract}

\begin{abstract}
The effect of aluminium in solid solution upon the thermal expansion coefficient of , pper has been measured for atomic concentrations between 1 and $7 \%$. The relative variation of the coefficient $\Delta \alpha / \alpha c=-1.1 \times 10^{-3}$ per atomic percent of aluminium has the same sign as the value $-3.8 \times 10^{-3}$ deduced by Cain and Thomas from measurements of 2 nd and 3rd order elastic constants, but it is three times smaller in absolute value.
\end{abstract}

1. Introduction. - On sait que la dilatation thermique d'un cristal, la variation de ses constantes élastiques avec la pression ou la température sont des manifestations de l'anharmonicité des forces interatomiques [1]. Des défauts cristallins, en faisant varier cette anharmonicité, doivent donc influencer les grandeurs ci-dessus et notamment la dilatation thermique. Nous avons pu ainsi récemment observer cet effet pour des défauts ponctuels d'irradiation dans l'aluminium et mesurer une dilatation intrinsèque de ces défauts [2]. Des mesures de dilatation ont aussi été décrites dans le cas d'alliages dilués [3].

Un cas nous a semblé intéressant, celui d'alliages dilués dont on connaisse par ailleurs les constantes élastiques d'ordre $3\left(C_{\alpha \beta}\right)$ et $\left(C_{\alpha \beta \gamma}\right)$. La connaissance de ces constantes donne en effet respectivement le terme harmonique et le premier terme anharmonique du développement de l'énergie élastique du cristal par rapport aux déformations $\varepsilon$ :

$$
U=U_{0}+\frac{1}{2} C_{\alpha \beta} \varepsilon_{\alpha} \varepsilon_{\beta}+\frac{1}{6} C_{\alpha \beta \gamma} \varepsilon_{\alpha} \varepsilon_{\beta} \varepsilon_{\gamma} .
$$

A l'aide d'un modèle du solide, ces données permettent de calculer la chaleur spécifique, le paramètre de Grüneisen $\gamma$ et le coefficient de dilatation thermique $\beta$. C'est ainsi qu'ayant mesuré les constantes élastiques d'ordre 2 et 3 d'une série d'alliages cuivre-aluminium de la phase $\alpha$ (concentration 3,$1 ; 7,4 ; 10,8 \%$ atomique d'aluminium), Cain et Thomas ont pu calculer pour chaque composition le paramètre de Grüneisen
$\gamma_{\mathrm{H}}$ dans la limite de haute température $\left(T>\theta_{\mathrm{D}}\right)$ et prévoir l'influence de l'aluminium en solution solide diluée sur le coefficient de dilatation thermique du cuivre [4]. Faute de données expérimentales, ils n'avaient pas pu vérifier ce dernier résultat. Nous présentons ici une mesure directe de l'effet de l'aluminium en solution solide sur la dilatation thermique du cuivre.

2. Expérience. - 2.1 ApPAREIL. - Nous utilisons le dilatomètre différentiel par interférométrie monté par Asty et Gilder [3]. On mesure les variations $\Delta \delta L$ de la différence de longueur $\delta L$ entre un échantillon d'alliage et un témoin de cuivre pur en fonction de la température entre 100 et $200 \mathrm{~K}$. Témoin et échantillon sont polycristallins et se présentent sous la forme de deux tubes de même axe, de même longueur $L=50 \mathrm{~mm}$, terminés à chaque extrémité par trois petits pieds définissant les plans de référence. Sur le témoin et sur l'échantillon reposent des lames optiques cylindriques à faces parallèles dont les plans en regard sont semi-réfléchissants. Ces plans formant un dièdre d'angle $\varepsilon$ faible $\left(10^{-3} \mathrm{rd}\right)$, un faisceau monochromatique $(\lambda=0,6 \mu)$ fourni par un laser $\mathrm{He}-\mathrm{Ne}$ y produit des franges d'interférence localisées qui apparaissent comme de fines raics noires sur fond clair d'interfrange $i=\lambda / 2 \varepsilon$. Une variation $\Delta \delta L$ de la différence de longueur entre témoin et échantillon, et donc de la distance entre plans réflecteurs se traduit par un 
déplacement proportionnel des franges que le montage optique, composé d'une lunette, d'un microscope, d'une caméra et d'un magnétoscope à longue durée d'enregistrement, permet de suivre en continu. La résolution obtenue est de $0,01 \mu$.

Le programme de température consiste en une suite de montées (ou descentes) en température à la vitesse de $2 \mathrm{~K} / \mathrm{h}$ pendant 2 à $4 \mathrm{~h}$, alternant avec des paliers de stabilisation de même durée, à la fin desquels sont relevés les points expérimentaux. La température de l'enceinte est alors stable à $0,01 \mathrm{~K}$ près pendant plusieurs dizaines de minutes.

2.2 PréParation Des ÉCHANTILlons. - Des lingots d'alliage ont été préparés par coulée sous ultravide au LETI (CEN-G) à partir de cuivre pur $(99,996 \%)$ dans les compositions suivantes : $0 \%$; $1 \% ; 3,1 \% ; 7,4 \%$ atomique d'aluminium. Nous n'avons pas fait d'analyse, mais selon le fabricant, l'erreur sur la composition serait de l'ordre de 0,1 à $0,2 \%$.

Le témoin (en cuivre pur) et les échantillons (dans chacune des quatre compositions) ont été usinés dans la masse, puis découpés chimiquement et recuits sous ultravide à $450^{\circ} \mathrm{C}$ pendant $4 \mathrm{~h}$. (La température de recuit, assez modérée a été choisie afin de comparer nos résultats avec ceux d'expériences en préparation sur des éléments d'alliage très volatils tels que $\mathrm{Ga}$, $\mathrm{Ge})$. Après avoir monté l'expérience, on laisse le dilatomètre se refroidir jusqu'à $100 \mathrm{~K}$ puis on effectue une montée suivie d'une descente en température entre 100 et $200 \mathrm{~K}$.

3. Résultats. - La figure 1 montre les courbes de dilatométrie différentielle obtenues pour les quatre compositions étudiées. Une détermination des pentes $B$ de ces courbes par les moindres carrés conduit aux valeurs données dans le tableau I. L'incertitude citée est égale à trois fois l'écart type estimé $\hat{\sigma}$. On voit que la pente $B_{0}$ obtenue dans l'expérience de zéro (dilatation différentielle entre le témoin et l'échantillon tous deux en cuivre pur) n'est pas tout à fait nulle. En admettant qu'elle traduit l'effet d'une erreur

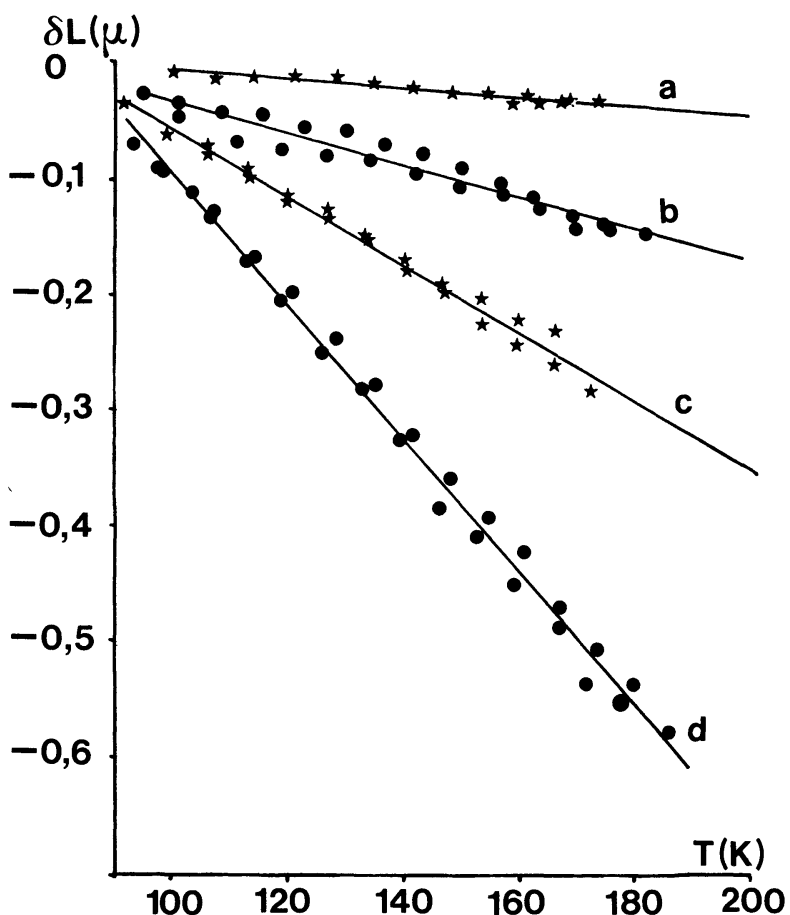

Fig. 1. - Différence de longueur entre l'échantillon en alliage $\mathrm{Cu}-\mathrm{Al}$ (concentration atomique $c$ ) et le témoin en $\mathrm{Cu}$ en fonction de la température. a) $c=0 \%$; b) $c=1 \%$; c) $c=3,1 \%$; d) $c=7,4 \%$.

[Length difference between a $\mathrm{Cu}-\mathrm{Al}$ alloy sample (atomic concentration $c$ ) and a pure $\mathrm{Cu}$ sample versus température. a) $c=0 \%$; b) $c=1 \%$; c) $c=3.1 \%$; d) $c=7.4 \%$.]

systématique, on corrige la pente et on estime la variation du coefficient de dilatation thermique pour chaque composition par la formule

$$
\Delta \alpha(c)=\frac{B(c)-B_{0}}{L} \text { où } L=50 \mathrm{~mm} .
$$

La dernière ligne du tableau I montre que $\Delta \alpha / c$ est constant dans la limite des erreurs expérimentales entre 1 et $7,4 \%$ atomique. Puisque dans le cuivre pur $\alpha=13 \times 10^{-6}$ à $T=140 \mathrm{~K}$ [5], température moyenne de nos mesures, la variation relative du coefficient de

Tableau I. - Effet de l'aluminium en solution solide sur la dilatation thermique du cuivre : B est la pente de la droite ajustée aux points expérimentaux par la méthode des moindres carrés, $\Delta \alpha / c$ la variation du coefficient de dilatation thermique linéaire par pour cent atomique d'aluminium.

[Effect of aluminium in solid solution upon the thermal expansion of copper : $B$ is the slope of the straight line best-fit to the experimental data, $\Delta \alpha / c$ the variation of the linear expansion coefficient per atomic percent of aluminium.]

Concentration atomique en $\mathrm{Al}(\%)$

$$
\begin{array}{cccc}
0 & 1 & 3,1 & , 7,4 \\
\overline{7} \pm 1,2 & -13, \overline{7} \pm 2,3 & -29 \overline{\overline{2}} \pm 2,4 & -56,0 \pm 2,2 \\
- & -2,0 \pm 0,7 & -1,7 \pm 0,2 & -1,4 \pm 0,1
\end{array}
$$$$
\text { Pente } B \times \overline{10}(\mu / \mathrm{K})
$$$$
\Delta \alpha / c \times 10^{8}\left(\mathrm{~K}^{-1} \text { par } \%\right.
$$$$
\text { atomique) }
$$ 
dilatation thermique du cuivre lorsqu'on ajoute de l'aluminium est

$$
\frac{\Delta \alpha}{\alpha c}=-\frac{1,4 \times 10^{-8}}{1,3 \times 10^{-5}}=-1,1 \times 10^{-3} \mathrm{~K}^{-1}
$$

par $\%$ atomique. Cain et Thomas prévoyaient $-3,8 \times 10^{-3} \mathrm{~K}^{-1}$. Leur estimation possède le bon signe (l'aluminium diminue l'anharmonicité de la matrice de cuivre, bien que l'aluminium pur ait un coefficient de dilatation supérieur à celui du cuivre), mais se trouve trois fois trop grande en valeur absolue. De nombreux facteurs peuvent être invoqués pour expliquer que l'accord ne soit pas très bon : le calcul de $\gamma$ est fait dans la limite $T>\theta_{\mathrm{D}}=344 \mathrm{~K}$ pour le cuivre; en outre, partant de la formule de MieGrüneisen $\beta=\gamma \chi C_{\mathrm{v}} / V$, Cain et Thomas sont obligés d'évaluer de nombreux termes :

$$
\frac{\mathrm{d} \beta}{\beta \mathrm{d} c}=\frac{\mathrm{d} \gamma}{\gamma \mathrm{d} c}+\frac{\mathrm{d} \chi}{\chi \mathrm{d} c}+\frac{\mathrm{d} C_{\mathrm{v}}}{C_{\mathrm{v}} \mathrm{d} c}-\frac{\mathrm{d} V}{V \mathrm{~d} c}
$$

de même ordre de grandeur mais de signes différents ; enfin l'utilisation de constantes élastiques pour représenter le spectre des phonons n'est vraiment justifiée qu'à très basse température.

Nous sommes heureux de remercier H. M. Gilder qui a attiré notre attention sur le travail de Cain et Thomas.

\section{Bibliographie}

[1] Leibfried, E., Ludwig, W., Solid State Phys. 12 (1961) 275.

[2] GANNe, J. P., Von Stebut, J., à paraître.

[3] Asty, M., Rapport CEA-R-4698 (1975).

[4] Cain, L. S., Thomas Jr., J. F., Phys. Rev. B 8 (1973) 5372.

[5] Kroeger, F. R., Swenson, C. A., J. Appl. Phys. 48 (1977) 853. 